УДК 519.2

\title{
Mathematical Discrete-Time Model of the Heterogeneous Integrated System of Corporate Information Management
}

\author{
Oleg Ja. Kravets* \\ Sustainable Developments in Civil Engineering Research Group \\ Faculty of Civil Engineering \\ Ton Duc Thang University \\ 19 Nguyen Huu Tho, District 7, 700 000, Ho Chi Minh City, \\ Vietnam
}

Received 10.02.2019, received in revised form 16.04.2019, accepted 06.06.2019

\begin{abstract}
The article considers some problems and peculiarities of the modelling and rational design of heterogeneous traffic servicing integrated systems. The aim of this paper is to develop the apparatus of modelling for distributed systems with a heterogeneous medium as part of a subsystem of managing the modelling and upgrading of the systems in question. The research subject is a system consisting of air and cable multiple-access systems. Protocol functions of every level are represented by a double-phase stochastic system within discrete time. Results of numerical experiments which were obtained in areas of high loads and over-loads allow us to determine possibilities for further prospective expansion of the system and to develop guidelines as to permissible values of system parameters for systems that are functioning in different modes. The obtained results can be used for the reconfiguration of both static and dynamic heterogeneous (according to the transmission medium) information systems.
\end{abstract}

Keywords: heterogeneous medium, communication system, mathematical model, optimal design. DOI: $10.17516 / 1997-1397-2019-12-5-560-570$.

\section{Introduction}

The paper continues previous study [1] and is dedicated to both construction of a model and evaluation of parameters of a heterogeneous integrated system of corporate information management based on the analysis of low-level protocols. The research subject is a system consisting of air and cable multiple-access systems.

The article [1] considers some problems and peculiarities of the modelling and rational design of heterogeneous traffic servicing integrated systems. In [1] a comparative analysis of existing technologies in modelling distributed systems with a heterogeneous medium as part of a subsystem of managing the modelling and upgrading of the systems in question done. Improvement in the management and modernization processes of the above-mentioned systems is prompted by the necessity to narrow down the range of admissible solutions to enhance the efficiency of the outlined processes and reduce their economic cost.

\section{Formalization of the object description}

Recall that the research object is a system consisting of air and cable multiple-access subsystems connected through an interface [2]. The coverage of the air subsystem is determined by the area of a circle of radius $R$. The cable subsystem link length is determined by the value $L$ (Fig. 1).

\footnotetext{
*olegkravets@tdt.edu.vn

(c) Siberian Federal University. All rights reserved
} 


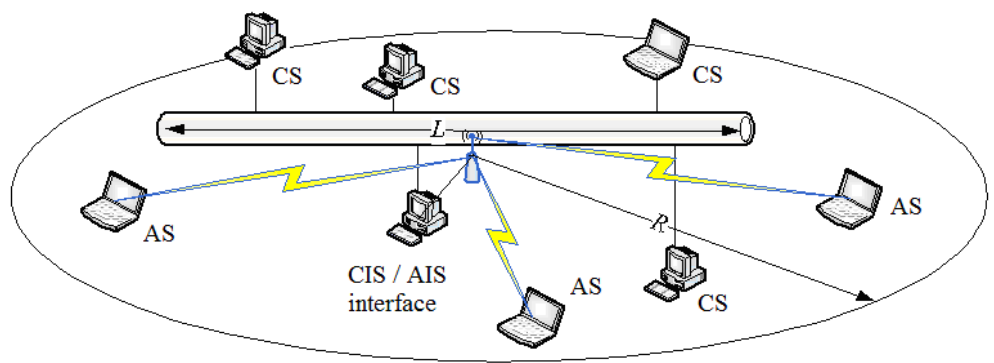

Fig. 1. Integrated information management system: CS - Cable Station; AS - Air Station; CIS - Cable Interface System; AIS - Air Interface System

Let us proceed toward development of a mathematical model of the observed system in discreet time, based on temporal diagrams and defined by them parameters of protocol blocks and time intervals of media occupancy during transmission of frames and transmission intervals of access management following on from [3].

The mathematical model is defined by z-transformation of the distribution series $[4,5]$ of delivery time $f_{q}(z)$ for messages of both types during their transmission from recipients of the air subsytem to recipients of the cable subsystem and vise versa, from recipients of the cable subsystem to recipients of the air subsystem.

\section{Development of the mathematical model}

Firstly, let us examine process of message transmission from recipients of the air subsystem to recipients of the cable subsystem, shown in the Fig. 2. Incoming delivery and servicing of message streams in the cable subsystem is performed on time intervals $T_{c}$, in the air subsystem $T_{a}$. Models of such systems belong to the class of discreet-stochastic models $[6,7]$ and can be modelled using mathematical apparatus of Markov chains and queuing systems [8].

Processes of message transmission from recipients of the air subsystem to recipients of the cable subsystem are modelled by two-phase queuing systems in discreet time.

Voice messages of the air subsystem, delivered to a recipient of the cable subsystem, are located in two phases: at the beginning in the air subsystem which is modelled as the queuing system in discreet time $M^{D} / G^{D} / 1$ containing $B_{a v}$ and later on in the cable subsystem which is modelled as the queuing system of the same type and containing $B_{i c v}$. Data messages, which are passing through the data subsystem also consequently reside in the first phase $\left(B_{a d}\right)$ and then in the second $\left(B_{i c d}\right)$. Voice and data messages of receiving stations of the air subsystem, addressed to recipients of the same subsystem, pass only through the first of abovementioned phases $\left(B_{a v}\right.$ and $\left.B_{a d}\right)$, as can be seen in the Fig. 2 .

Z-transformation of distribution series (z-tds) of delivery time for data and voice messages in two-phase queueing systems is defined as $f_{q a c \varepsilon}(z), \varepsilon=\overline{v, d}$ during information transfer from the air subsystem into the cable subsystem; $f_{a \varepsilon}(z), \varepsilon=\overline{v, d}$ denotes z-tds of delivery time of voice messages and data in the air subsystem with an interval of $T_{a}$ and $f_{\text {qice }}(z), \varepsilon=\overline{v, d}$ represents the z-tds of delivery time for voice messages and data to clients of the cable subsystem in the second phase with an interval of $T_{k}$. Then following relation takes place:

$$
f_{q a c \varepsilon}(z)=f_{q a \varepsilon}(z) * f_{\text {qice }}(z), \quad \varepsilon=\overline{v, d}
$$

here symbol $*$ signifies consequent connection of phases: the first during the time interval $T_{a}$ and the second during the interval $T_{c}$. In case when $T_{a}=T_{c}$ symbol $*$ signifies multiplication. 


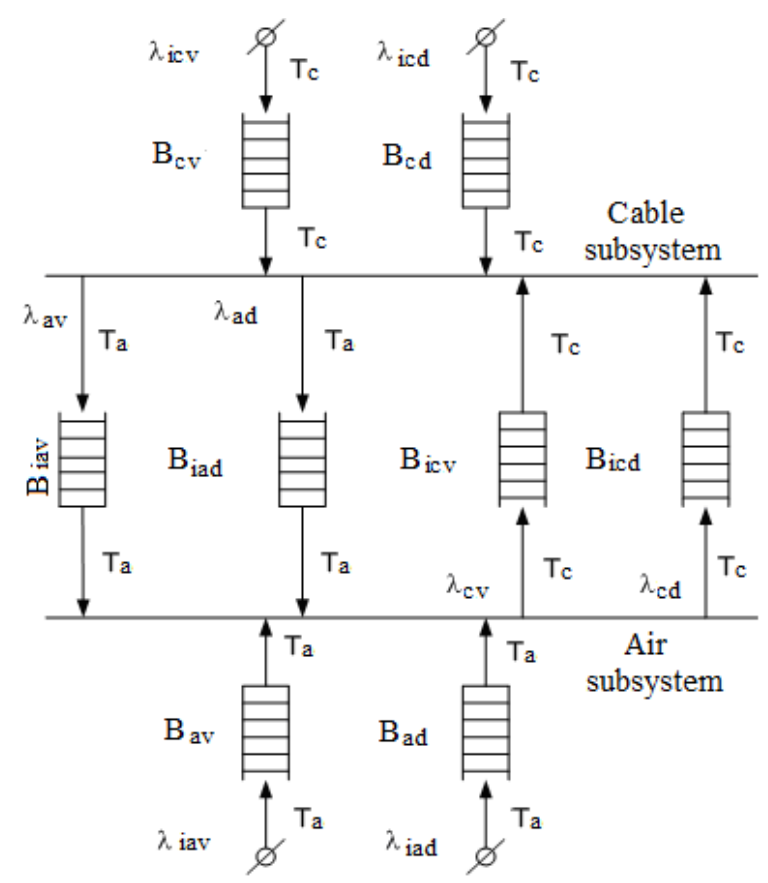

Fig. 2. Stochastic representation of the model of interaction between the air and the cable communication subsystems

\subsection{Parameters of the air subsystem}

Let us proceed to determination of components of (1). According to access and transmission protocols in the air subsystem $[9,10]$, z-tds of residence time of voice messages or data on intervals $T_{a}$ :

$$
\begin{aligned}
& f_{q a \varepsilon}(z)=\frac{\left(1-\Theta_{a \varepsilon}\right)(1-z) g_{a \varepsilon}(z)}{1-\left(1-q_{i a \varepsilon}\right) z-q_{i a \varepsilon} z g_{a \varepsilon}(z)}, \quad \varepsilon=\overline{v, d}, \quad \Theta_{a \varepsilon}<1, \\
& q_{i a \varepsilon}=\lambda_{i a \varepsilon} T_{a}, \quad \lambda_{i a \varepsilon} T_{a}<1,
\end{aligned}
$$

where $g_{a \varepsilon}$ is z-tds of service interval of voice messages or data,

$$
g_{a \varepsilon}(z)=g_{a a \varepsilon}\left(g_{s a \varepsilon}^{-1}(z)\right) .
$$

When the CFE (crucial feedback with expectations [11,12]) algorithm is used, z-tds of a number of transmissions of voice messages in the air subsystem is defined as:

$$
g_{a a d}(z)=Q_{s a d} /\left(z-P_{s a d}\right)
$$

where $P_{\text {sad }}$ is a probability of a loss of data messages in the air subsystem and is represented by the expression:

$$
P_{\text {sad }}=1-Q_{\text {sad }}, \quad Q_{\text {sad }}=\left(1-p_{a}\right)^{n_{c a d}}, \quad n_{c a d}=r_{a d}+r_{y}+r_{c a}+k_{d},
$$

where $p_{a}$ is a probability of an error in the air medium of transmission, $n_{c a d}$ is a parameter of the protocol of the air subsystem.

z-tds of a number of transmissions during servicing of a single voice message will be defined by:

$$
g_{a a v}(z)=z^{-1} \text {. }
$$


Numbers $n_{a \varepsilon}$ of recipients that have voice messages and data in the area of coverage of transmitting-and-receiving station are discreet random numbers whereas maximal quantity of stations of voice messages and data recipients is, respectively, $N_{a v}$ and $N_{a d}$. Then conditional $z$-tds of the one-time transmission interval of a voice message is written as:

$$
\begin{aligned}
& g_{s_{a v}}\left(z \mid n_{a v}=N_{a v} ; n_{a d}=N_{a d}\right)= \\
& =z^{-c_{a v}}\left(\overline{\Theta_{a v}} z^{-c_{o a}}+\Theta_{a v} z^{-c_{a v}}\right)^{N_{a v}}\left(\overline{\Theta_{a d}} z^{-c_{o a}}++\Theta_{a d} z^{-c_{a d}}\right)^{N_{a d}} \bullet \\
& \cdot\left(\overline{\Theta_{i a v}} z^{-c_{o a}}+\Theta_{i a v} z^{-c_{a v}}\right)\left(\overline{\Theta_{i a d}} z^{-c_{o a}}+\Theta_{i a d} z^{-c_{a d}}\right), \\
& \overline{\Theta_{i a v}}=1-\Theta_{i a v}, \quad \overline{\Theta_{i a d}}=1-\Theta_{i a d},
\end{aligned}
$$

where $N_{a v}$ and $N_{a d}$ are fixed values of discrete stochastic values $n_{a v}$ and $n_{a d}$, respectively; $c_{a v}, c_{o a}$ and $\mathrm{M}$ are parameters of the air subsystem protocols; $\Theta_{i a v}, \Theta_{i a d}-$ probabilities that buffers $B_{i a v}$ and $B_{i a d}$ are being occupied.

For two-dimensional binomial distribution of parameters $n_{a v}$ and $n_{a d}$ :

$$
\varphi\left(n_{a v}, n_{a d}\right)=C_{N_{a v}}^{n_{a v}} \rho_{v t}^{n_{a v}}\left(1-\rho_{v t}\right)^{N_{a v}-n_{a v}} \cdot C_{N_{a d}}^{n_{a d}} \rho_{d t}^{n_{a d}}\left(1-\rho_{d t}\right)^{N_{a d}-n_{a d}},
$$

where $\rho_{v t}$ is a voice transmission parameter, $\rho_{d t}$ is a data transmission parameter.

When the rule of conditional mathematical expectation is applied, after averaging over $n_{a v}$ and $n_{a d}$ unconditional z-tds of a service interval for voice messages can be inferred:

$$
\begin{aligned}
& g_{s_{a v}}(z)=z^{-c_{a v}}\left(1-\rho_{v t}\left(1-\overline{\Theta_{a v}} z^{-c_{o a}}-\Theta_{a v} z^{-c_{a v}}\right)\right)^{N_{a v}^{-1}} \bullet \\
& \cdot\left(1-\rho_{d t}\left(1-\overline{\Theta_{a d}} z^{-c_{o a}}-\Theta_{a d} z^{-c_{a d}}\right)\right)^{N_{a d}} \bullet \\
& \cdot\left(\overline{\Theta_{i a v}} z^{-c_{o a}}+\Theta_{i a v} z^{-c_{a v}}\right)\left(\overline{\Theta_{i a d}} z^{-c_{o a}}+\Theta_{i a d} z^{-c_{a d}}\right) .
\end{aligned}
$$

When session models are used for transmission of voice messages [6] with the parameter $\rho_{a}$ (specific recipient load of voice messages), z-tds of the one-time interval for servicing of a voice message on intervals $T_{a}$ after averaging over a random number of recipients in the air subsystem is defined by the expression:

$$
\begin{aligned}
& g_{s_{a v}}(z)=z^{-c_{a v}}\left(1-\rho_{a} \rho_{v t}\left(1-\overline{\Theta_{a v}} z^{-c_{o a}}-\Theta_{a v} z^{-c_{o v}}\right)\right)^{N_{a v}^{-1}} \bullet \\
& \cdot\left(1-\rho_{d t}\left(1-\overline{\Theta_{a d}} z^{-c_{o a}}-\Theta_{a d} z^{-c_{a d}}\right)\right)^{N_{a d}} \bullet \\
& \cdot\left(\overline{\Theta_{i a v}} z^{-c_{o a}}+\Theta_{i a v} z^{-c_{a v}}\right)\left(\overline{\Theta_{i a d}} z^{-c_{o a}}+\Theta_{i a d} z^{-c_{a d}}\right) .
\end{aligned}
$$

Let us similarly define z-tds of one-time transmission interval of data messages on intervals $T_{a}$ in the air subsystem. Conditional z-tds of one-time transmission interval during servicing of data messages is written as:

$$
\begin{aligned}
& g_{s_{a d}}\left(z \mid n_{a v}=N_{a v} ; n_{a d}=N_{a d}\right)=z^{-c_{a d}}\left(\overline{\Theta_{a d}} z^{-c_{o a}}+\Theta_{a d} z^{-c_{a d}}\right)^{N_{a d}^{-1}} \bullet \\
& \cdot\left(\overline{\Theta_{a v}} z^{-c_{o a}}+\Theta_{a v} z^{-c_{a v}}\right)^{N_{a v}} \bullet\left(\overline{\Theta_{i a d}} z^{-c_{o a}}+\Theta_{i a d} z^{-c_{a d}}\right) \bullet\left(\overline{\Theta_{i a v}} z^{-c_{o a}}+\Theta_{i a v} z^{-c_{a v}}\right) .
\end{aligned}
$$

After averaging with usage of expression (8) unconditional z-tds of one-time service interval for data messages can be found:

$$
\begin{aligned}
& g_{s_{a d}}(z)=z^{-c_{a d}}\left(1-\rho_{d t}\left(1-\overline{\Theta_{a d}} z^{-c_{o a}}-\Theta_{a d} z^{-c_{a d}}\right)\right)^{N_{a d}^{-1}} \bullet \\
& \cdot\left(1-\rho_{v t}\left(1-\overline{\Theta_{a v}} z^{-c_{o a}}-\Theta_{a v} z^{-c_{a v}}\right)\right)^{N_{a v}} \cdot\left(\overline{\Theta_{i a v}} z^{-c_{o a}}+\Theta_{i a v} z^{-c_{a v}}\right) \bullet \\
& \boldsymbol{\bullet}\left(\overline{\Theta_{i a d}} z^{-c_{o a}}+\Theta_{i a d} z^{-c_{a d}}\right) .
\end{aligned}
$$


For the session model of the source of data messages z-tds of the one-time service interval of data messages is given by the expression:

$$
\begin{aligned}
& g_{s_{a d}}(z)=z^{-c_{a d}}\left(1-\rho_{d t}\left(1-\overline{\Theta_{a d}} z^{-c_{o a}}-\Theta_{a d} z^{-c_{a d}}\right)\right)^{N_{a d}^{-1}} \bullet \\
& \bullet\left(1-\rho_{a} \rho_{v t}\left(1-\overline{\Theta_{a v}} z^{-c_{o a}}-\Theta_{a v} z^{-c_{a d}}\right)\right)^{N_{a v}} \bullet \\
& \bullet\left(\overline{\Theta_{i a v}} z^{-c_{o a}}+\Theta_{i a v} z^{-c_{a v}}\right)\left(\overline{\Theta_{i a d}} z^{-c_{o a}}+\Theta_{i a d} z^{-c_{a d}}\right)
\end{aligned}
$$

\subsection{Traffic parameters between subsystems}

Voice messages in the cable subsystem are transmitted inside the session, which is conducted with a specific recipient load $\rho_{c}$ on intervals $T_{c}$ in the binomial stream with the probability $q_{i c v}$ and intensity $\lambda_{i c v}=q_{i c v} / T_{c}$ and in the air subsystem - with a specific recipient load $\rho_{a}$ on intervals $T_{a}$ with probability $q_{a v}$ and intensity $\lambda_{a v}$, expression for which will be obtained further. Traffic of the air subsystem recipients for voice messages and data is closed into itself within the subsystem with probabilities $\nu_{a v}$ and $\nu_{a d}$, respectively.

Then for the second phase of (1) considering working access protocol z-tds of residence time of voice and data messages in the Integrated Information Management System (IIMS) during information transmission from the air subsystem into the cable subsystem on intervals $T_{c}$ (buffers $B_{i c d}$ and $B_{i c v}$ in the Fig. 2) are defined as follows:

$$
\begin{aligned}
& f_{q i c \varepsilon}(z)=\frac{1-\Theta_{i c \varepsilon}(1-z) g_{i c \varepsilon}(z)}{1-\left(1-q_{c \varepsilon)} z-q_{c \varepsilon} z g_{i c \varepsilon}(z)\right.}, \\
& \varepsilon=\overline{v, d} ; \Theta_{i c \varepsilon}<1, \quad q_{c \varepsilon}=\lambda_{c \varepsilon} T_{c}, \lambda_{c \varepsilon} T_{c}<1 .
\end{aligned}
$$

After previous assumptions about type of traffic and transmission protocol of voice messages have been introduced, the intensity of a stream of this type of messages which enters the buffer $B_{i c v}$ (Fig. 2) of the interface station from recipients of the air subsystem to recipients of the cable subsystem is written as:

$$
\lambda_{c v}=\lambda_{i a v} N_{a v}\left(1-\nu_{a v}\right) \overline{P_{c a v}} \rho_{v d} \rho_{a}, \overline{P_{c a v}}=1-P_{c a v},
$$

where $\rho_{a}$ is a specific recipient load of voice messages in the air subsystem; $P_{c a v}$ is a probability of voice messages loss:

$$
P_{c a v}=1-Q_{c a v}, \quad Q_{c a v}=\left(1-p_{a}\right)^{n_{\text {cav }}}, \quad n_{c a v}=r_{a v}+r_{y}+r_{v d}+k_{v} .
$$

Intensity of the voice message stream which enters the buffer $B_{i c d}$ (Fig. 2) IIMS during transmission from the air subsystem in the cable subsystem is as follows:

$$
\lambda_{d t}=\lambda_{i a d} N_{a d}\left(1-\nu_{a d}\right) \rho_{d t}
$$

where $\rho_{v t}$ nad $\rho_{d t}$ are probabilities of appearance of recipients of voice and data in the air subsystem. z-tds of a service interval for messages of both types in the second phase of (1) on intervals $T_{c}$ is found from the expression:

$$
g_{i c \varepsilon}(Z)=g_{a c \varepsilon}\left(g_{\text {sice }}^{-1}(z)\right)
$$

\subsection{Parameters of the cable subsystem}

z-tds of a number of recurrent transmissions in the cable subsystem during servicing of a single data message and using the CFE algorithm is defined as:

$$
\begin{aligned}
& g_{a c d}(z)=Q_{c c d} /\left(z-P_{c c d}\right), \\
& P_{c c d}=1-Q_{c c d}, \quad Q_{c c d}=\left(1-p_{c}\right)^{n_{c c d}},
\end{aligned}
$$


where $P_{c c d}$ is a probability of data messages loss in the cable subsystem; $Q_{c c d}$ is a probability of servicing a request; $p_{c}$ is a probability of an error in cable transmission medium; the parameter $n_{c c d}$ of the cable subsystem protocol can be calculated using following expression:

$$
n_{c c d}=r_{a d}+r_{y}+r_{c}+k_{d} .
$$

During transmission of voice messages in erase-mode (i.e. a one-time transmission) z-tds of a number of transmissions will be described by the expression:

$$
g_{a c v}(z)=z^{-1} \text {. }
$$

Let us define z-tds of a one-time transmission interval of voice and data-like messages in the cable subsystem. Z-tds of the one-time transmission interval during servicing of voice messages for the binomial model of voice sources [13] in the cable subsystem on discreet intervals $T_{c}$ is written as:

$$
\begin{aligned}
& g_{s_{i c v}}(z)=z^{-c_{c v}}\left(\overline{\Theta_{c v}} z^{-c_{o c}}+\Theta_{c v} z^{-c_{c v}}\right)^{N_{c v}} \bullet \\
& \bullet\left(\overline{\Theta_{c d}} z^{-c_{o c}}+\Theta_{c d} z^{-c_{c d}}\right)^{N_{c d}}\left(\overline{\Theta_{i c d}} z^{-c_{o c}}+\Theta_{i c d} z^{-c_{c d}}\right)
\end{aligned}
$$

and for the session model:

$$
\begin{aligned}
& g_{s_{i c v}}(z)=z^{-c_{c v}}\left(1-\rho_{a c}\left(\overline{\Theta_{c v}} z^{-c_{o c}}+\Theta_{c v} z^{-c_{c v}}\right)\right)^{N_{c v}} \bullet \\
& \bullet\left(\overline{\Theta_{c d}} z^{-c_{o c}}+\Theta_{c d} z^{-c_{c d}}\right)^{N_{c d}} *\left(\overline{\Theta_{i c d}} z^{-c_{o c}}+\Theta_{i c d} z^{-c_{c d}}\right),
\end{aligned}
$$

where $\overline{\Theta_{i c d}}=1-\Theta_{i c d}$.

When we introduce the parameter $\alpha(z)$ which equals:

$$
\alpha(z)=\left(1-\rho_{c}\left(1-\overline{\Theta_{c v}} z^{-c_{o c}}+\Theta_{c v} z^{-c_{c v}}\right)\right)^{N_{c v}} \cdot\left(\overline{\Theta_{c d}} z^{-c_{o c}}+\Theta_{c d} z^{-c_{c d}}\right)^{N_{c d}}
$$

the expression for z-tds of one-time service of voice messages can be obtained:

$$
g_{s_{i c v}}(z)=z^{-c_{c v}}\left(\overline{\Theta_{i c v}} z^{-c_{o c}}+\Theta_{i c d} z^{-c_{c d}}\right) \alpha(z)
$$

where $c_{o c}, c_{c v}$ and $c_{c d}$ are parameters of the cable subsystem protocols. z-tds of one-time transmission interval of a message during service of data messages for the binomial model of data sources in the cable subsystem on discreet intervals $T_{c}$ is given by:

$$
\begin{aligned}
& g_{s_{i c d}}(z)=z^{-c_{c d}}\left(\overline{\Theta_{c v}} z^{-c_{o c}}+\Theta_{c v} z^{-c_{c v}}\right)^{N_{c v}} \bullet \\
& \bullet\left(\overline{\Theta_{c d}} z^{-c_{o c}}+\Theta_{c d} z^{-c_{c d}}\right)^{N_{c d}}\left(\overline{\Theta_{i c d}} z^{-c_{c d}}+\Theta_{i c v} z^{-c_{c v}}\right)
\end{aligned}
$$

and for the session model (considering previously introduced notation $\alpha(z)$ ) the expression is written as:

$$
g_{s_{i c d}}(z)=z^{-c_{c d}}\left(\overline{\Theta_{i c v}} z^{-c_{o c}}+\Theta_{i c v} z^{-c_{c v}}\right) \alpha(z),
$$

where $\overline{\Theta_{i c v}}=1-\Theta_{i c v}$.

\section{Models of data transmission between subsystems}

Let us examine transmission processes for both types of messages directed from recipients of the cable to recipients of the air subsystem that are modelled by two-phase queuing systems in discreet time.

As in previous examination, during transmission of voice messages and data from recipients of the cable subsystem to recipients of the air subsystem, messages are passing through two phases. Let us denote z-tds of delivery time in these phases as $f_{q a c \varepsilon}(z), \varepsilon=\bar{v}, d$. During transmission of 
information within the cable subsystem z-tds of residence time of voice messages and data can be denoted as $f_{q c \varepsilon}(z), \varepsilon=\overline{v, d}$. Hence, in studied process of delivery z-tds of residence time for messages of both types can be written as following expression:

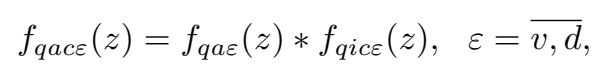

where $f_{\text {qia }}(z), \varepsilon=\overline{v, d}$ is z-tds of transmission time for voice messages and data in the second phase with the interval $T_{a}$ (in IIMS); sign * denotes sequential connection of the first on the interval $T_{c}$ and the second on the interval $T_{a}$ phases.

Processes of message exchange between recipients of the cable subsystem are written as $f_{q c \varepsilon}$ on intervals $T_{c}$ and for the air subsystem - as $f_{q a \varepsilon}$ on intervals $T_{a}$, correspondingly. The component $f_{\text {qace }}$ in above-mentioned relation conditionally reflects residence time of messages in two-phase systems with different intervals.

z-tds of delivery time for messages during their transmission from the cable onto the air subsystem is defined as following:

$$
\begin{aligned}
& f_{q c \varepsilon}(z)=\frac{\left(1-\Theta_{c \varepsilon}\right)(1-z) g_{c \varepsilon}(z)}{1-\left(1-q_{i c \varepsilon}\right) z-q_{i c \varepsilon} z g_{c \varepsilon}(z)}, \quad \varepsilon=\overline{v, d}, \quad \Theta_{c \varepsilon}<1, \\
& q_{i c \varepsilon}=\lambda_{i c \varepsilon} T_{c}, \quad \lambda_{i c \varepsilon} T_{c}<1,
\end{aligned}
$$

where $g_{c \varepsilon}(z)$ is a z-tds of servicing interval for either voice messages or data:

$$
g_{c \varepsilon}(z)=g_{a c \varepsilon}\left(g_{s c \varepsilon}^{-1}(z)\right) .
$$

z-tds of a number of repeated transmissions of data messages $g_{a c d}(z)$ is derived from the expression (19) and z-tds of one-time transmission interval of voice messages $g_{a c v}(z)$ is obtained from the formula (20).

In case of the binomial source of voice messages, z-tds of a one-time transmission interval of voice messages is written as:

$$
\begin{aligned}
& g_{s_{c v}}(z)=z^{-c_{c v}}\left(\overline{\Theta_{c v}} z^{-c_{o c}}+\Theta_{c v} z^{-c_{c v}}\right)^{N_{c v}} \bullet \\
& \cdot\left(\overline{\Theta_{c d}} z^{-c_{o c}}+\Theta_{c d} z^{-c_{c d}}\right)^{N_{c d}}\left(\overline{\Theta_{i c v}} z^{-c_{o c}}+\Theta_{i c v} z^{-c_{c v}}\right) \\
& \boldsymbol{\bullet}\left(\overline{\Theta_{i c d}} z^{-c_{o c}}+\Theta_{i c d} z^{-c_{c d}}\right) .
\end{aligned}
$$

When the session model of the source of voice messages with a specific recipient load $\rho_{a c}$ is used, z-tds of one-time transmission interval is defined as:

$$
\begin{aligned}
& g_{s_{i c v}}(z)=z^{-c_{c v}}\left(1-\rho_{a c}\left(\overline{\Theta_{c v}} z^{-c_{o c}}+\Theta_{c v} z^{-c_{c v}}\right)\right)^{N_{c v}-1} \bullet\left(\overline{\Theta_{c d}} z^{-c_{o c}}+\Theta_{c d} z^{-c_{c d}}\right)^{N_{c d}} \bullet \\
& \bullet\left(\overline{\Theta_{i c v}} z^{-c_{o c}}+\Theta_{i c v} z^{-c_{c v}}\right)\left(\overline{\Theta_{i c d}} z^{-c_{o c}}+\Theta_{i c d} z^{-c_{c d}}\right) .
\end{aligned}
$$

z-tds of a one-time transmission interval of data message in case of the binomial source of data messages of the cable subsystem on discreet intervals $T_{c}$ is inferred from:

$$
\begin{aligned}
& g_{s_{c d}}(z)=z^{-c_{c d}}\left(\overline{\Theta_{c v}} z^{-c_{o c}}+\Theta_{c v} z^{-c_{c v}}\right)^{N_{c v}}\left(\overline{\Theta_{c d}} z^{-c_{o c}}+\Theta_{c d} z^{-c_{c d}}\right)^{N_{c d}-1} \bullet \\
& \cdot\left(\overline{\Theta_{i c v}} z^{-c_{o c}}+\Theta_{i c v} z^{-c_{c v}}\right)\left(\overline{\Theta_{i c d}} z^{-c_{o c}}+\Theta_{i c d} z^{-c_{c d}}\right) .
\end{aligned}
$$

In case of the session model same expression takes on following form:

$$
\begin{aligned}
& g_{s_{c d}}(z)=z^{-c_{c d}}\left(1-\rho_{a c}\left(\overline{\Theta_{c v}} z^{-c_{o c}}+\Theta_{c v} z^{-c_{c v}}\right)\right)^{N_{c v}}\left(\overline{\Theta_{c d}} z^{-c_{o c}}+\Theta_{c d} z^{-c_{c d}}\right)^{N_{c d}-1} \bullet \\
& \boldsymbol{\bullet}\left(\overline{\Theta_{i c v}} z^{-c_{o c}}+\Theta_{i c v} z^{-c_{c v}}\right)\left(\overline{\Theta_{i c d}} z^{-c_{o c}}+\Theta_{i c d} z^{-c_{c d}}\right) .
\end{aligned}
$$


Voice messages in the air subsystem are transmitted within a session which is conducted with a specific recipient load $\rho_{a e}$ on discreet intervals $T_{a}$ in the binomial stream with the probability $q_{i a v}$ and intensity $\lambda_{i a v}=q_{i a v} / T_{a}$. Traffic of the cable subsystem recipients is closed into itself in the subsystem with probabilities $\nu_{c v}$ and $\nu_{c d}$ according to voice and data messages.

Then z-tds of delivery time of an $\varepsilon$-type message on intervals $T_{a}$ in IIMS during transmission of messages from the cable subsystem into the air subsystem ( $B_{\text {iav }}$ and $B_{\text {iad }}$ in the Fig. 2$)$ is written as:

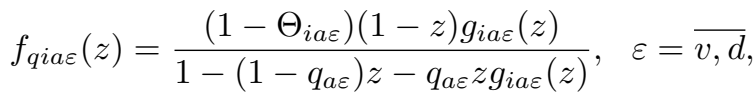

$$
\begin{aligned}
& \text { where: } \Theta_{i a \varepsilon}<1 \quad q_{a \varepsilon}=\lambda_{a \varepsilon} T_{a} \quad \lambda_{a \varepsilon} T_{a}<1 .
\end{aligned}
$$

Intensity of a stream of voice messages incoming in the buffer $B_{\text {iav }}$ (Fig. 2) IIMS from cable to air recipients is defined as follows:

$$
\lambda_{a v}=\lambda_{i c v} N_{c v}\left(1-\nu_{c v}\right) \overline{P_{c v}} \rho_{c}, \quad \overline{P_{c v}}=1-P_{c v}
$$

where $\rho_{c}$ is a specific recipient load of voice messages in the cable subsystem; $P_{c v}$ is a probability of loss of voice messages in the cable subsystem:

$$
P_{c v}=1-Q_{c v}, \quad Q_{c v}=\left(1-p_{c}\right)^{n_{c v}}
$$

whereas $n_{c v}$ can be derived from the following expression: $n_{c v}=r_{a v}+r_{y}+r_{c}+k_{v}$. Intensity of data messages stream which is entering in the buffer $B_{i a d}$ IIMS from cable to air recipients is determined by following expression:

$$
\lambda_{a d}=\lambda_{i c d} N_{c d}\left(1-\nu_{c d}\right),
$$

where $\nu_{c v}$ and $\nu_{c d}$ are probabilities of traffic closures for voice messages and data, correspondingly, in the cable subsystem; $\lambda_{i c v}=q_{i c v} / T_{c}$ is an intensity of voice message streams which enter into IIMS (i.e. into the buffer $B_{i a d}$ ).

In the formula (34) z-tds of service interval for messages of both types is defined as:

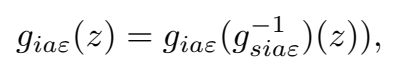

where expressions for z-tds of a number of transmissions of voice messages and data are represented by formulas (4) and (6), respectively.

Expressions for z-tds of one-time transmission intervals during service of voice messages and data for the binomial model of the source of voice messages and data, respectively, in the air subsystem on discreet intervals $T_{a}$ are given by:

$$
\begin{aligned}
& g_{s_{i a v}}(z)=z^{-c_{a v}}\left(\overline{\Theta_{a v}} z^{-c_{o a}}+\Theta_{a v} z^{-c_{a v}}\right)^{N_{a v}} \bullet \\
& \cdot\left(\overline{\Theta_{a d}} z^{-c_{o a}}+\Theta_{a d} z^{-c_{a d}}\right)^{N_{a d}}\left(\overline{\Theta_{i a d}} z^{-c_{o a}}+\Theta_{i a d} z^{-c_{a d}}\right), \\
& g_{s_{i a d}}(z)=z^{-c_{a d}}\left(\overline{\Theta_{a v}} z^{-c_{o a}}+\Theta_{a v} z^{-c_{a v}}\right)^{N_{a v}} \bullet \\
& \bullet\left(\overline{\Theta_{a d}} z^{-c_{o a}}+\Theta_{a d} z^{-c_{a d}}\right)^{N_{a d}}\left(\overline{\Theta_{i a v}} z^{-c_{o a}}+\Theta_{i a v} z^{-c_{a d}}\right),
\end{aligned}
$$

and for the session model of the source of voice messages and data:

$$
\begin{gathered}
g_{s_{i a v}}(z)=z^{-c_{a v}}\left(1-\rho_{a} \rho_{v t}\left(\overline{\Theta_{a v}} z^{-c_{o a}}+\Theta_{a v} z^{-c_{a v}}\right)\right)^{N_{a v}} \bullet \\
\bullet\left(1-\rho_{d t}\left(\overline{\Theta_{a d}} z^{-c_{o a}}+\Theta_{a d} z^{-c_{a d}}\right)\right)^{N_{a d}}\left(\overline{\Theta_{i a d}} z^{-c_{o a}}+\Theta_{i a d} z^{-c_{a d}}\right), \\
g_{s_{i a d}}(z)=z^{-c_{a d}}\left(1-\rho_{a} \rho_{v t}\left(\overline{\Theta_{a v}} z^{-c_{o a}}+\Theta_{a v} z^{-c_{a v}}\right)\right)^{N_{a v}} \bullet \\
\bullet\left(1-\rho_{d t}\left(\overline{\Theta_{a d}} z^{-c_{o a}}+\Theta_{a d} z^{-c_{a d}}\right)\right)^{N_{a d}}\left(\overline{\Theta_{i a d}} z^{-c_{o a}}+\Theta_{i a d} z^{-c_{a d}}\right) . \\
-567-
\end{gathered}
$$


In outlined expressions values of probability of buffers being occupied can be found from following system of interference equations:

$$
\begin{aligned}
& \Theta_{a \varepsilon}=q_{i a \varepsilon} \overline{n_{s a \varepsilon}} ; \quad \overline{n_{s a \varepsilon}}=\left.\left(d / d z^{-1}\right) g_{a \varepsilon}(z)\right|_{z=1} ; \quad \varepsilon=\overline{v, d} \\
& \Theta_{c \varepsilon}=q_{i c \varepsilon} \overline{n_{s c \varepsilon}} ; \quad \overline{n_{s c \varepsilon}}=\left.\left(d / d z^{-1}\right) g_{c \varepsilon}(z)\right|_{z=1} ; \quad \varepsilon=\overline{v, d} \\
& \Theta_{i c \varepsilon}=q_{c \varepsilon} \overline{n_{s i c \varepsilon}} ; \quad \overline{n_{s i c \varepsilon}}=\left.\left(d / d z^{-1}\right) g_{i c \varepsilon}(z)\right|_{z=1} ; \quad \varepsilon=\overline{v, d}
\end{aligned}
$$

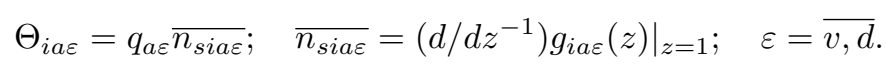

The process of calculation of occupied state probabilities of station buffers consists in solving the system of linear equations by using Gaussian elimination.

\section{Results of numerical modelling}

Performed modelling of voice messages and data transmission between subsystems of twophase queuing systems in discreet time enabled us to obtain corresponding expressions that describe z-transformation of distribution series for the service interval of voice messages and data for the session model of the message source $g(z)$ which was modelled. In addition, we determined probabilities of occupied conditions of station buffers which are contained in abovementioned expressions and can be calculated from the system of interference equations (43). The mathematical model of heterogeneous integral communication system is specified by expressions which were formulated above.

The numerical experiment was carried out in order to examine influence of exploitation parameters on functioning of the system and its both temporal and probabilistic characteristics. Results of these studies which were obtained in regions of high loads and over-loads allow us to define possibilities of further prospective expansion of the system and to develop guidelines for permissible values of parameters for systems, which work in different modes. It was determined that the air subsystem is subjected to the biggest influence during change of exploitation parameters. The air subsystem makes the largest influence on the system in comparison with the cable subsystem. Fig. 3 depicts an example of dependence of average time variation of a delivery time within the system and subsystems from the number of stations $\mathrm{N}$.

As it can be inferred from the presented plot, the air subsystem is subjected to the biggest influence. The region of a working load is located within the limit of 100 users during the increase of the number of users in this subsystem. At the same time, the cable subsystem is able to support normal mode of functioning even when number of users increases up to 140-200.

\section{Conclusions}

The authors have used the methodology of conceptual and mathematical modelling within the queuing systems theory. The article presents a model of heterogeneous subsystem representation, assigns the optimal task for choosing the multicriteria of a system building variants, and proposes integrated protocols for the functioning of the system as well as a mathematical model based on z-transferring of delivery time and service interval distribution series.

Functions of each level protocols are represented as a single-phase stochastic system within discrete time. Definition algorithms expressing the average times for messages to be delivered are developed.

Session and binomial models of data and voice sources have been constructed for both air and cable subsystems. Models have been created for data transmission processes between subsystems which utilize two-phase queuing systems in discreet time. 


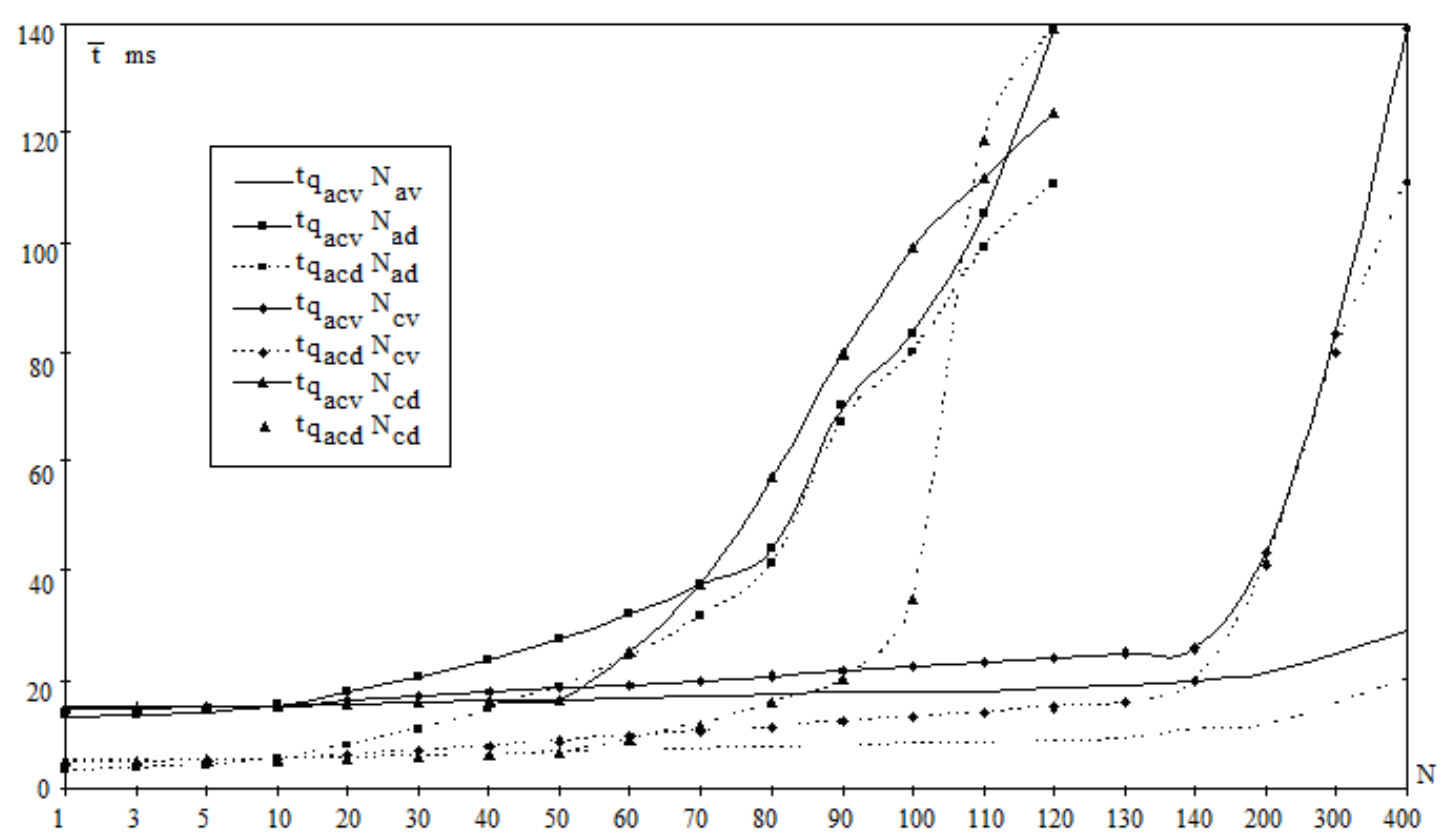

Fig. 3. Dependencies of average delivery time $\bar{t}$ from the number of stations $\mathrm{N}$

Expressions were obtained for distributions of holding time for voice messages and data; for the service interval of either voice messages or data; for the number of data messages' transmissions; for the interval of one-time transmission of voice messages; for the interval of one-time transmission of voice-type messages and data in the cable subsystem; for delivery time of a message during message transmission from the cable to the air subsystems.

Results of quantitative studies have been obtained in regions of both high-loads and overloads. They enable us to determine possibilities of future prospective expansion of the system as well as to develop guidelines for permissible values of system parameters for systems which function in different modes. It was determined that in comparison with the cable subsytem the air subsystem is heavily subjected to the influence introduced by a change of operating parameters.

The obtained results can be used both for static and dynamic heterogeneous (according to the transmission medium) information system reconfiguration.

\section{References}

[1] O.Ja.Kravets, O.N.Choporov, The problems and peculiarities of modelling integrated systems of heterogeneous traffic services, J. Sib. Fed.l Univ.. Math. Phys., 11(2018), 5, 581-587.

[2] Q-Y.Han, J.Li, A semantic integration mode for heterogeneous system, IEEE Computer Design and Applications (ICCDA), 2010, DOI: 10.1109/ICCDA.2010.5541213.

[3] O.Ja.Kravets, Mathematical Modeling of Parameterized TCP Protocol, Automation and Remote Control, 74(2013), no. 7, 1218-1224.

[4] G.S.Mudholkar, Y.P.Chaubey, On the Distribution of Fisher'S Transformation of the Correlation Coefficient, Communication in Statistics - Simulation and Computation, 5(1976), no. 4, 163-172. 
[5] D.C.Rao, Joint Distribution of z Transformations Estimated from the Same Sample, Hum Hered, 29(1979), 334-336.

[6] P.T.Artikis, Discrete stochastic models and global information risk treatment operations in strategic processes, Journal of Discrete Mathematical Sciences and Cryptography, 20(2017), no. $2,477-491$.

[7] F.V.Louveaux, Discrete stochastic location models, Ann Oper Res, 6(1986), 21.

[8] B.Filipowicz, J.Kwiecien, Queuing systems and networks. Models and applications, Bulletin of the Polish Academy of Sciences. Technical Sciences, 56(2008), no. 4, 379-390.

[9] Y.Takaki, M.Ando, K.Maesako, et al., Efficient and reliable packet transfer protocol for wireless multihop bidirectional communications, International Journal of Distributed Sensor Networks, 14(2018), no. 1.

[10] P.Sethi, Internet of Things: Architectures, Protocols, and Applications, Journal of Electrical and Computer Engineering, 2017, Article ID 9324035, DOI:10.1155/2017/9324035.

[11] F.Usta, Z.Eren, H.Kurtaran, Crashworthiness Optimization of Nested and Concentric Circular Tubes Using Response Surface Methodology and Genetic Algorithm, Latin American Journal of Solids and Structures, 15(2018), no. 5.

[12] J.G.Brookshear, D.T.Smith, D.Brylow, Computer science: an overview, Addison-Wesley, 2012.

[13] I.Atanasov, Modeling Aspects of Autonomous Smart Metering Information System, International Journal on Information Technologies and Security, 8(2016), no. 1, 3-18.

\section{Математическая модель гетерогенной интегральной корпоративной информационно-управляющей системы в Дискретном времени}

Олег Я. Кравец

Строительный факультет Университет Тон Дюк Тханг 19 Нгуен Хуу То, округ 7, Хошимин, 700000 Вьетнам

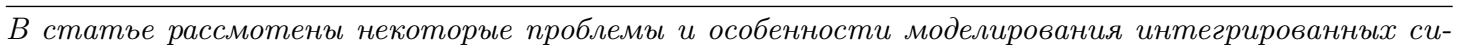
стем обслуживания гетерогенного трабика. Цель статьи - развитие аппарата моделирования распределенных систем с гетерогенной средой как части подсистемы управления моделированием и модернизацией. Предметом исследования является система, состоящая из воздушных и кабелъных систем множественного доступа.

Функиии протоколов каждого уровня представлены двухфазными стохастическими системами в дискретном времени.

Результаты численных исследований, полученные в областях высоких загрузок и перегрузок, позволяют определить возможности дальнейшего расширения системь, а также разработать рекомендации по допустимым величинам параметров систем, работающих в различных режимах. Полученные результаты могут быть исполъзованы для реконфигурации как статических, так и динамических гетерогенных (по передающей среде) информационных систем.

Ключевые слова: гетерогенная среда, система связи, математическая модель, оптималъное проектирование. 